\title{
Proof of concept review
}

\section{Panitumumab: the evidence of its therapeutic potential in metastatic colorectal cancer care}

\author{
Erika Martinelli ${ }^{1}$, Floriana Morgillo ${ }^{1}$, Teresa Troiani1 ${ }^{1}$, Giampaolo Tortora ${ }^{2}$, Fortunato Ciardiello ${ }^{1}$ \\ ${ }^{1}$ Cattedra di Oncologia Medica, Dipartimento Medico-Chirurgico di Internistica Clinica e Sperimentale \\ "F. Magrassi e A. Lanzara", Seconda Università degli Studi di Napoli, Napoli, Italy; ${ }^{2}$ Dipartimento \\ di Endocrinologia ed Oncologia Molecolare e Clinina, Università di Napoli Federico II, Napoli, Italy
}

\begin{abstract}
Introduction: Colorectal cancer is the fourth most common malignant disease. Of newly diagnosed patients, $40 \%$ have metastatic disease at diagnosis, and approximately $25 \%$ of patients with localized disease at diagnosis will ultimately develop metastatic disease. The benefits of systemic chemotherapy in the treatment of metastatic colorectal cancer over best supportive care have been established. Panitumumab (ABX-EGF) is the first fully human monoclonal antibody developed for use in colorectal cancer that targets the extracellular domains of epidermal growth factor receptor.

Aims: The goal of this article is to review the published evidence for the use of panitumumab in the treatment of metastatic colorectal cancer to define its therapeutic potential.

Evidence review: The major evidence of panitumumab activity in colorectal cancer has appeared in meeting report abstracts. One phase II study in monotherapy, one in combination with chemotherapy, and one phase III study have included only patients with metastatic colorectal cancer.

Clinical potential: To date, in phase II clinical studies panitumumab has demonstrated antitumor activity in advanced, refractory colorectal cancer. As monotherapy it resulted in a 10\% response rate with $38 \%$ of patients having stable disease, and a $36 \%$ response rate with $46 \%$ stable disease when combined with chemotherapy. A phase III study indicates a clinically significant advantage of panitumumab as third-line monotherapy over best supportive care. Panitumumab appears to have a good tolerability profile, with no maximum tolerated dose yet defined.
\end{abstract}

Core Evidence. 2007;2(2):81-88.

Key words: ABX-EGF, human monoclonal antibody, metastatic colorectal cancer, panitumumab

\section{Core evidence proof of concept summary for panitumumab in metastatic colorectal cancer}

\begin{tabular}{|ll|}
\hline Outcome measure & Emerging evidence \\
\hline Efficacy & $\begin{array}{l}\text { Potential to use as monotherapy (at a dose of } 6 \mathrm{mg} / \mathrm{kg} \text { every } 3 \text { weeks) or in combination with chemotherapy } \\
\text { (at a dose of } 2.5 \mathrm{mg} / \mathrm{kg} \text { once per week); stable disease achieved in up to } 46 \% \text { of patients, and progression-free survival } \\
\text { of } 2-10.9 \mathrm{months}\end{array}$ \\
$\begin{array}{l}\text { Evidence of activity with a response rate between } 10 \% \text { and } 36 \% \\
\text { Response rates }\end{array}$ & $\begin{array}{l}\text { Even in studies where immunohistochemistry expression of EGFR was required, it does not correlate with objective response } \\
\text { Good toxicity profile; no maximum tolerated dose has been reported, and no differences in pharmacokinetic parameters } \\
\text { between the once weekly, every } 2 \text { weeks, and every } 3 \text { weeks dosage schedules }\end{array}$ \\
\hline EGFR, epidermal growth factor receptor. & \\
\hline
\end{tabular}




\section{Scope, aims, and objectives}

Colorectal cancer is the fourth most common malignant disease (IARC 2002; Meyerhardt \& Mayer 2005) and the second leading cause of cancer death in the USA (Jemal et al. 2005; Meyerhardt \& Mayer 2005). Even though different therapeutic strategies with new drugs have doubled the median overall survival (OS) of metastatic colorectal cancer patients up to 21.5 months, it remains less than 2 years. Active salvage options are clearly needed for this disease.

Panitumumab (ABX-EGF) is the first fully human monoclonal antibody that targets the extracellular domains of epidermal growth factor receptor (EGFR) to be investigated in colorectal cancer. The objective of this review is to evaluate the potential role of panitumumab in metastatic colorectal cancer.

\section{Methods}

The English language medical literature was searched for relevant articles related to the use of panitumumab in metastatic colorectal cancer. The search terms used were "Panitumumab or ABX-EGF," "colorectal cancer" and the articles published covered a period between 1988 to November 2006. The following databases were used:

- PubMed, http://www.ncbi.nlm.nih.gov/entrez

- National Cancer Institute, http://www.cancer.gov

- Clinical Evidence (BMJ), http://www.clinicalevidence.com

- Clinical Trials, http://www.clinicaltrials.com

The annual scientific sessions from the American Society of Clinical Oncology (ASCO), European Society of Medical Oncology (ESMO), American Association of Cancer Research (AACR), and ASCO Gastrointestinal Cancers Symposium (ASCO Gl) were searched for relevant meeting abstracts. A total of 19 articles were identified, 11 of those represented by systematic review. Nine articles were excluded because they were considered not relevant (Table 1). A further paper was identified (Van Cutsem et al. 2007) replacing two earlier abstracts.

Few studies were identified for panitumumab use specifically in colorectal cancer; this indication was however investigated in early trials in which the drug was used in a variety of solid tumors. No health economics publications were found.

\section{Disease overview}

Colorectal cancer is one of the most common malignancies. The worldwide incidence estimated in 2002 was 1023152 (550 465 men, 472687 women) (IARC 2002). Of newly diagnosed patients, $40 \%$ have metastatic disease at diagnosis, and approximately $25 \%$ of patients with localized disease at diagnosis will ultimately develop metastatic disease. With the exception of patients with localized liver metastases, which is potentially curable by surgical treatment, the majority of patients with
Table 1 | Evidence base included in the review

\begin{tabular}{|lcc|}
\hline Category & \multicolumn{2}{c|}{ Number of records } \\
\cline { 2 - 3 } & Full papers & Abstracts \\
\hline Initial search & 19 & 11 \\
records excluded & 9 & 3 \\
records included & 10 & 8 \\
Additional studies identified & 1 & 0 \\
Level 1 clinical evidence & 0 & 2 \\
Level 2 clinical evidence & 2 & 5 \\
Level $\geq 3$ clinical evidence & 9 & 1 \\
trials other than RCT & 0 & 0 \\
case reports & 0 & 0 \\
Economic evidence & 0 & 0 \\
\hline $\begin{array}{l}\text { For definition of levels of evidence, see Editorial lnformation on inside back cover. } \\
\text { RCT, randomized controlled trial. }\end{array}$ & & \\
\hline
\end{tabular}

metastatic colorectal cancer die of their disease.

The essential element of the etiology of colorectal cancer is a process of genetic change in the epithelial cells of the colonic mucosa (Vogelstein et al. 1988). Epidemiologic factors have provided initial evidence about specific factors that initiate the process of carcinogenesis in large bowel mucosa (Winawer \& Shike 1992). Chief among the factors that can initiate colorectal cancer development are a predisposition to mutagen effects, fecal mutagens, meat intake, bile acids, altered vitamin and mineral intake, and fecal $\mathrm{pH}$. Investigators are most suspicious of the role of diet in causation of colorectal cancer (Peters at al. 1992).

The incidence of the disease has been slowly declining over the past few decades, probably because of improved screening and earlier detection of premalignant disease. Colorectal cancer lends itself to screening because of the long period between the development of early mucosal abnormalities and the development of invasive carcinoma. Adenomatous polyps are the welldescribed precursor lesions of invasive colorectal cancer and can be effectively managed by endoscopic intervention. In the general population, the risk of development of a colorectal adenoma is approximately $19 \%$, and it is estimated that $2-5 \%$ of these sporadic polyps will develop into an invasive carcinoma (Ransohoff \& Lang 1991). Unfortunately, adherence to screening recommendations by individuals at average risk is suboptimal, and despite the ability to prevent colorectal cancer this disease remains a major cause of morbidity and mortality in western society.

\section{Current therapy options}

The treatment options for colorectal cancer depend on disease stage at diagnosis. In locally advanced disease (stage I, II, III) surgery is the most effective intervention. Even in cases of 
metastatic colorectal carcinoma, surgery may be required for palliation. The benefits of systemic chemotherapy in the treatment of metastatic colorectal cancer have become established in the past 20 years, and there is good evidence of improved quality of life and OS (Simmonds 2000).

Fluorouracil has been considered the standard of care in the past decade used both in advanced and in metastatic disease. It has been used with and without biomodulating agents such as leucovorin and methotrexate, alone as an intravenous bolus, by high-dose weekly 24-hour infusions, or by protracted continuous infusions which offer a higher response rate (33\% vs $14 \%, P=0.0004$ ) (de Gramont et al. 1997). However, progression-free survival (PFS) differs only modestly, being 5 weeks longer with continuous infusion $(P=0.001)$ and there is no statistical benefit in terms of OS (de Gramont et al. 1997).

Capecitabine provides an oral alternative to intravenous fluorouracil in both the metastatic and adjuvant setting. In two separate phase III trials, capecitabine has demonstrated superior response rate, equivalent PFS and OS, and decreased toxicity compared with bolus fluorouracil/leucovorin (Hoff et al. 2001; Van Cutsem et al. 2004).

The modern era of colorectal cancer chemotherapy began in the mid-1990s when two novel chemotherapeutic agents-irinotecan and oxaliplatin - were found to have significant activity in metastatic colorectal cancer. The introduction of irinotecan and oxaliplatin has nearly doubled the median survival of patients with metastatic disease, replacing fluorouracil/folinic acid as the standard of care. The consequence of the combination regimens using irinotecan (Saltz et al. 2000) oxaliplatin (de Gramont et al. 2000) and fluorouracil/folinic acid has been to increase the efficacy of first-line therapy from response rates of about $20 \%$, a time to progression of 4 months and a median OS of approximately 10 months in 1995, to response rates of between $35 \%$ and $>50 \%$, a median time to progression in the region of 8 months, and median OS of up to 21.5 months in 2004 (Tournigand et al. 2004). Although the combination of oxaliplatin/fluorouracil/folinic acid (FOLFOX) demonstrated significantly higher efficacy compared with irinotecan/fluorouracil bolus/leucovorin (IFL), oxaliplatin/folinic acid (FOLOX) and irinotecan/fluorouracil bolus + continuous infusion leucovorin (FOLFIRI) appear to be equivalent in terms of efficacy. A phase III trial comparing FOLFIRI and FOLFOX6 (oxaliplatin/fluorouracil/folinic acid) with crossover at progression, found that response rate $(56 \%$ vs $54 \%$ ), PFS ( 8.5 vs 8.1 months), and OS (21.5 vs 20.6 months) were nearly identical (Tournigand et al. 2004).

Recently, targeted therapy has presented the opportunity to improve on the efficacy of current treatments without exacerbating the associated toxicity; the advent of monoclonal antibodies has added improvements in survival and costs in metastatic colorectal cancer. Attention has focused on EGFR and on vascular endothelial growth factor (VEGF), which is implicated in angiogenesis.

Angiogenesis is the process of new blood vessel formation and it is considered critical for tumor growth. Unlike traditional chemotherapy, angiogenesis inhibitors specifically target the formation of new blood vessels without interfering with dividing hematopoietic stem cells or gastrointestinal cells. At the time of writing, bevacizumab (Avastin ${ }^{\circledR}$ ), a humanized antibody directed against VEGF, has been approved in the USA and in Europe for first-line therapy in metastatic colorectal cancer. Approval was based on a phase III pivotal trial that evaluated the activity of bevacizumab $5 \mathrm{mg} / \mathrm{kg}$ every 2 weeks when added to IFL, the standard care in the USA. Significant differences in PFS (10.6 vs 6.2 months) and OS (20.6 vs 15.6 months) were registered in the bevacizumab-containing arm. Improvement in response rate ( $45 \%$ vs $35 \%$ ) was moderate, maybe because the angiogenesis inhibitors mainly result in tumor growth inhibition rather than regression (Hurwitz et al. 2004). The Eastern Cooperative Oncology Group (ECOG) trial E3200 has evaluated the activity of bevacizumab in combination with oxaliplatin and fluorouracil as second-line therapy. The final results, presented at the ASCO meeting 2005, showed an advantage (12.5 vs 10.7 months) in terms of OS, with a reduced hazard ratio for death of 0.73 (Giantonio et al. 2005).

Another potential target in colorectal cancer treatment is EGFR, a cell membrane growth factor receptor with tyrosine kinase activity which plays a crucial role in the control of key cellular transduction pathways in both normal and cancer cells. The EGFR is overexpressed in a variety of human tumors, including colorectal, head and neck, breast, lung, prostate, kidney, pancreas, ovary, brain, and bladder cancers (Woodburn 1999; Mendelsohn \& Baselga 2000). Functional activation of EGFR is due to the formation of either EGFR-EGFR homodimers or of heterodimers with one of the other three members of the EGFR family of growth factor receptors (HER-2, HER-3, and HER-4) following binding of an EGFR-selective ligand, such as EGF, transforming growth factor alfa (TGF-alfa), or amphiregulin. This results in conformational changes that allow activation of the EGFR tyrosine kinase with the phosphorylation of specific tyrosine residues within the EGFR intracellular carboxyl-terminal domain. Phosphorylated tyrosine residues serve as docking sites for several signaling proteins that initiate a complex intracellular signal transduction cascade that ultimately affects regulation of gene transcription (Yarden 2001; Yarden \& Sliwkowski 2001). The activity of EGFR could be neutralized by monoclonal antibodies that bind to the extracellular domain and competitively inhibit ligand binding to prevent receptor dimerization and subsequent activation of the receptor tyrosine kinase. The EGFR-monoclonal antibody complex is internalized, causing a transient decrease in EGFR expression (Ciardiello \& Tortora 2001). Eighty percent of colorectal cancers are EGFR-positive by immunohistochemistry staining (Cunningham et al. 2004). This parameter does not correlate with clinical response and, moreover, even EGFR immunohistochemistry-negative tumors could respond to EGFR inhibitor treatment (Chung et al. 2005).

Cetuximab $\left(\right.$ Erbitux $\left.^{\circledR}\right)$ is a chimeric human monoclonal antibody against EGFR, approved worldwide in metastatic colorectal cancer refractory to irinotecan-based therapy. Cetuximab is typically administered at $400 \mathrm{mg} / \mathrm{m}^{2}$ initially, followed by $250 \mathrm{mg} / \mathrm{m}^{2}$ weekly thereafter. The first phase II nonrandomized trials attested to its 
activity as monotherapy (response rate 11\%, stable disease 35\%) and in combination with IFL (response rate 19\%, stable disease $27 \%$ ) in patients who had failed prior irinotecan therapy (Saltz et al. 2001, 2004). These results were confirmed in a large, randomized, phase III trial carried out in 329 patients, of whom 218 received cetuximab plus irinotecan and 111 cetuximab alone (Cunningham et al. 2004). The response rate with the combination was significantly higher than with monotherapy (23\% vs $11 \%$, $P=0.007$ ). Disease control rates were $56 \%$ and $32 \%$, respectively, and time to progression was also significantly greater for the combination arm (4.1 vs 1.5 months, $P<0.001)$. The median survival time was 8.6 months in the cetuximab arm $(P=0.48)$.

The role of cetuximab combined with FOLFOX4 (oxaliplatin/fluorouracil/leucovorin) in a first-line setting has been tested in a phase II trial of 43 patients. A total of $9 \%$ of patients achieved a complete remission and $63 \%$ had partial response, with an overall confirmed response rate of $72 \%$. Ten patients underwent liver metastasectomy, the overall response rate was $81 \%$, and the PFS was 10.2 months (Diaz Rubio et al. 2005). More information about the role of EGFR inhibition as a first-line option in colorectal cancer should come from the Crystal study, a large multicenter study in which 1212 patients with previously untreated metastatic colorectal cancer expressing EGFR have been randomized to receive FOLFIRI with or without cetuximab; preliminary data are expected in 2007.

These interesting results have led to investigation of the combination between EGFR inhibitors and antiangiogenic agents. The preliminary results of a randomized phase II study in patients with irinotecan-refractory, metastatic colorectal cancer have been reported. In this trial, named the BOND-2 study, 39 patients were treated with the combination of cetuximab, irinotecan, and bevacizumab, and 35 patients received cetuximab plus bevacizumab treatment. The authors have reported a $37 \%$ response rate with a time to progression of 7.9 months in the triple-drug combination, compared with $20 \%$ and 5.6 months in patients treated with cetuximab plus bevacizumab (Saltz et al. 2005).

Targeted therapy represents a new frontier in treatment of colorectal cancer. New compounds such as panitumumab could have the same efficacy as cetuximab with lower incidence of hypersensitivity reactions due to it being a fully human antibody.

\section{Outcomes achieved in clinical development}

Panitumumab is being evaluated in clinical trials as both a monotherapy and in combination with other agents for the treatment of various types of cancer including colorectal, lung, and kidney.

Several phase II studies with panitumumab have been carried out, and other phase III trials are ongoing. An important difference compared with cetuximab is that panitumumab is a fully human Ig2 monoclonal antibody created by Abgenix's XenoMouse technology, which creates antibodies that contain no murine protein (Mendez et al. 1997). The goal of this technique is to offer effective high-affinity therapy with a minimum of allergic reactions or anaphylaxis.

\section{Monotherapy}

Until now, 1130 patients have been treated with panitumumab as monotherapy, including 789 with metastatic colorectal cancer, 229 of whom have been enrolled in a phase III trial. The remaining 341 patients had renal cell carcinoma and other solid tumors.

The phase I data of safety, pharmacokinetics, and antitumor activity in solid tumors were presented at the ASCO meeting 2005 (Weiner et al. 2005). In this study sequential cohorts of patients were enrolled to receive four infusions at different dose levels and schedules of panitumumab monotherapy ranging from 0.01 to $5 \mathrm{mg} / \mathrm{kg}$ once per week, to $6 \mathrm{mg} / \mathrm{kg}$ once every 2 weeks, or $9 \mathrm{mg} / \mathrm{kg}$ once every 3 weeks. Patients were prescreened for EGFR expression by immunohistochemistry and had to have at least $1+$ (defined as low staining intensity) expression in $\geq 10 \%$ of tumor cells. A total of 96 patients were enrolled; tumor types included 39 colorectal, 21 prostate, 15 renal, 14 nonsmall cell lung (NSCLC), three pancreatic, three esophageal/gastroesophageal, and one anal cancer. Pharmacokinetic exposure was similar between $2.5 \mathrm{mg} / \mathrm{kg}$ per week, $6 \mathrm{mg} / \mathrm{kg}$ every 2 weeks, and $9 \mathrm{mg} / \mathrm{kg}$ every 3 weeks.

By investigator assessment, five patients had a partial response, all of whom had colon cancer and 19 had stable disease including eight with colorectal cancer, five with NSCLC, three with prostate cancer, two with renal cancer, and one with gastroesophageal cancer. The incidence of skin-related toxicities was dose dependent and reached a plateau at $>2.0 \mathrm{mg} / \mathrm{kg}$ once per week. Grade 3 or 4 related adverse events were noted in $10 \%$ of patients with grade 3 skin-related effects being the most frequent ( $7 \%$ of patients). No maximum tolerated dose was reached, and no human antihuman antibody formation or infusion-related reactions was observed.

A phase II open-label trial presented at the ASCO meeting 2004 (Hecht et al. 2004; Malik et al. 2005) assessed the efficacy of panitumumab monotherapy in patients with metastatic colorectal cancer who failed therapy with a fluoropyrimidine, fluorouracil ( \pm leucovorin), and either irinotecan or oxaliplatin. A total of 150 patients were enrolled and received at least one dose of panitumumab; 148 were included in the safety and efficacy analysis. Patients were subdivided into two cohorts according to immunohistochemistry tumor staining. Cohort $A$ included patients with EGFR 2+ (defined as moderate staining intensity by immunohistochemistry) or $3+$ (defined as high staining intensity immunohistochemistry) in $\geq 10 \%$ tumor cells; cohort $B$ included patients with EGFR of the sum of $1+, 2+$, and $3+$ in $\geq 10 \%$ of tumor cells, but with the sum of $2+$ and $3+$ in $<10 \%$ of tumor cells. Panitumumab was given intravenously at $2.5 \mathrm{mg} / \mathrm{kg}$ once weekly in 8-week cycles; patients continued treatment until disease progression or unacceptable toxicity. Panitumumab monotherapy resulted in a $10 \%$ response rate with stable disease in 38\% of patients; the median duration of response was 5.2 months 
[95\% confidence interval (Cl) 4.5, 7.5 months]. The median PFS was 2 months (95\% Cl 1.9, 3.8 months), and the median survival amounted to 7.9 months ( $95 \% \mathrm{Cl} 5.7,9.9$ months). The four most frequently reported grade 3 or 4 treatment-related adverse effects were rash, fatigue, vomiting, nausea, and pruritus. Skin-related adverse events were reported in 141 patients (95\%; 5\% grade 3 and no grade 4). One infusion-related grade 3 adverse event was registered but no dose modification was required. No human antihuman antibodies were observed. A relationship between skin rash severity and survival seems to be similar to that noted with the use of cetuximab (Hecht et al. 2006b). Moreover, its efficacy appears to be similar in patients with both low and negative EGFR levels (Hecht et al. 2006a, 2007).

This has been confirmed by a multicenter, single-arm, phase II study that enrolled patients with low (defined as 1-9\% of tumor membrane staining by immunohistochemistry) or negative $(<1 \%$ tumor membrane staining) EGFR levels. Patients had failed 2-3 prior regimens containing an adequate dose of fluoropyrimidine, irinotecan, and oxaliplatin; they received panitumumab at the dose of $6 \mathrm{mg} / \mathrm{kg}$ every 2 weeks. Tumor assessments were taken from week 8 until disease progression and were performed by an independent eligibility review committee. The interim analysis included 89 patients, $57 \%$ of whom were male, and with a median age of 61 years. Six of thirty-five $(2 \%)$ partial responses were reported in patients with low expression of EGFR $(<1 \%)$ and $4 / 51$ $(8 \%)$ in patients with negative levels of EGFR, and stable disease occurred in $11 / 35(31 \%)$ versus $15 / 51$ (29\%), with a disease control rate of $37 \%$ versus $39 \%$. Time to progression was 9 weeks for both low or negative EGFR patients, with duration of response of 31 versus 20 weeks, and PFS of 7 versus 8 weeks. The most common adverse events were dermatitis acneiform (72\% in total, $6 \%$ grade $3 / 4)$, erythema $(69 \%, 6 \%)$, pruritus $(65 \%$, $4 \%)$, and hypomagnesemia (53\%, 10\%). Three patients (<3\%) had an infusion reaction per investigator (one grade 3 infusion reaction). The overall response rate was judged the same in the local and in the central review committee (Hecht et al. 2007).

The activity of panitumumab has also been tested in patients with metastatic colorectal cancer expressing $\geq 10 \%$ EGFR. Patients with documented disease progression during or following adequate doses of fluoropyrimidine, irinotecan, and oxaliplatin regimens received panitumumab $6 \mathrm{mg} / \mathrm{kg}$ every 2 weeks until disease progression. Study endpoint was objective response rate (ORR) at week 16. In the interim analysis, 91 enrolled patients received more than one dose of panitumumab. The efficacy set consisted of 23 male and 16 female patients, median age 58.6, 95\% ECOG score $\leq 1,74 \%$ with colon cancer, and $26 \%$ with rectal cancer. At week 16 , three patients $(8 \%)$ had a partial response, eight $(21 \%)$ had stable disease, and 19 (49\%) had disease progression. Response durations were 12.4, 13.2, and 14.0 weeks. As main adverse events skin toxicity was reported in $96 \%$ of the cases, $8 \%$ for nail and $5 \%$ for eye toxicity, $27 \%$ for diarrhea, and $12 \%$ for hypomagnesemia. One patient had a grade 3 hypersensitivity reaction related to panitumumab (Berlin et al. 2006).

A large, phase III, multicenter, pivotal trial (study 408) has randomized patients with oxaliplatin and irinotecan-refractory
EGFR-expressing metastatic colorectal cancer between best supportive care (BSC) and BSC plus panitumumab at a dose of $6 \mathrm{mg} / \mathrm{m}^{2}$ every 2 weeks (Van Cutsem et al. 2007). The aim of this pivotal trial was to show a significant difference in PFS. A total of 1040 patients were screened, of whom only 463 were randomized (1:1); 231 received panitumumab with BSC, 232 received BSC only, 174 patients received panitumumab under crossover protocol. Patients were stratified for sex, age, ECOG performance status, number of metastatic sites, prior chemotherapy, and intensity of EGFR staining. Panitumumab showed a $46 \%$ decrease in tumor progression rate versus BSC alone (hazard ratio $0.54 ; 95 \% \mathrm{Cl} 0.44$, 0.66; $P<0.000000001$, stratified log-rank test). The subset analyses demonstrated consistent treatment effect of panitumumab in all subgroups of patients. The response rate was significantly higher in the panitumumab arm, with $8 \%$ partial response rate and $28 \%$ achieving stable disease, compared with no partial responses and $10 \%$ stable disease with BSC alone. The time to response was 8 weeks, and the median duration of the response was 17 weeks. Panitumumab also showed activity in crossover study patients with $10 \%$ achieving partial response and $32 \%$ stable disease.

Skin reactions of any grade occurred in $90 \%$ of patients receiving panitumumab, and in $9 \%$ of those receiving BSC; the incidence of grade 3-4 skin-related adverse reactions was $14 \%$ and $0 \%$ for panitumumab and BSC, respectively. Skin reactions consisted of dermatitis acneiform $7 \%$, erythema $5 \%$, pruritus $2 \%$, and rash $1 \%$.

Overall, $33 \%$ of patients in the panitumumab arm experienced grade 3 adverse effects, mainly due to abdominal pain ( $23 \%$ vs $17 \%$ with BSC alone), fatigue ( $24 \%$ vs $15 \%$ ), nausea ( $22 \%$ vs $15 \%$ ), diarrhea ( $21 \%$ vs $11 \%$ ), and $2 \%$ of all patients experienced grade 4 toxicity. Hypomagnesemia occurred in $38 \%$ of patients treated with panitumumab versus $2 \%$ of those in the BSC arm; the patients with grade 3-4 toxicity (3\% in both groups) received intravenous magnesium. The incidence of potential infusion reactions was low with no grade 3-4 reactions. Only one grade 2 hypersensitivity reaction was reported and treatment was discontinued (Van Cutsem et al. 2007).

Recently, data have been presented from the exploratory efficacy analyses from this trial of PFS, ORR, and OS in patient subsets based on baseline performance status (ECOG score 0-1 vs 2-3) and age ( $<65$ vs $\geq 65$ years). Most had ECOG score of $0-1(87 \%$ panitumumab, $84 \% \mathrm{BSC}$ ); $42 \%$ of panitumumab patients and $39 \%$ of the BSC group were aged $\geq 65$ years. The treatment effect on PFS favored panitumumab regardless of age or ECOG status. Among the panitumumab patients, similar PFS, OS, and ORR were seen between elderly and younger patients and within both ECOG status groups (Van Cutsem et al. 2007).

\section{Combination therapy}

There are also data showing good activity as first-line treatment of metastatic colorectal cancer when panitumumab $2.5 \mathrm{mg} / \mathrm{kg}$ once weekly is combined with chemotherapy. These data are from a two-part phase II study, in the first part of which panitumumab is added to IFL. 
Of 19 patients, $47 \%$ had a response (complete or partial) and disease was stable in $26 \%$, with a median PFS of 5.6 months and median OS of 16.8 months (Berlin et al. 2004). The rate of early disease progression was slow and was experienced in only one patient; all patients who responded to the panitumumab combination developed skin rash. The second part of this study enrolled patients with the same characteristics $(n=24)$ but they received panitumumab with FOLFIRI as first-line chemotherapy. The primary endpoint of the study was the incidence of diarrhea; the secondary endpoints included ORR, PFS, and OS. Thirty-three percent of patients achieved partial response and $46 \%$ had stable disease, with a PFS of 10.9 months. The median OS was not reached because at the time of analysis (May 2005) 23 of 24 patients were still alive (Hecht et al. 2006b). The incidence of grade 3 and 4 diarrhea was $53 \%$ and $5 \%$, respectively, for part 1 of the study, and $25 \%$ and $0 \%$, respectively, in part 2 . Treatment-related skin reactions were present in both parts, reaching grade 3 severity in $16 \%$ of patients in part 1 and in $17 \%$ of patients in part 2; there were no grade 4 reactions. Other grade 3 treatment-related adverse events included hypokalemia (16\%), fatigue (11\%), and nausea $(5 \%)$ in part 1; none of these events occurred in part 2. Grade 4 treatment-related hypomagnesemia occurred in one patient in each part of the study. There were no severe infusion reactions, or human antihuman antibody formation (Berlin et al. 2004; Hecht et al. 2006b).

The activity of panitumumab in combination with FOLFIRI and AMG706 was tested by Burris and colleagues (2007). AMG706 is an oral, multikinase inhibitor with antiangiogenic activity that selectively targets VEGF, PDGF (platelet-derived growth factor), and Kit receptors. In this ongoing phase I study panitumumab was given at a dosage of $6 \mathrm{mg} / \mathrm{kg}$ by intravenous infusion every 2 weeks, and AMG706 was given continuously from day 3 of cycle 1 in escalating doses; patients also received either FOLFOX or FOLFIRI based on prior systemic therapy. Dose-limiting toxicity was assessed during the first two cycles, and tumor response was evaluated every 6-8 weeks from week 6 . At the time of writing, data are available only for the cohort containing FOLFIRI. Twenty-two patients were enrolled and had received at least one dose of AMG706 at the time of data cut-off (50 mg, n=12; $75 \mathrm{mg}$, $\mathrm{n}=7 ; 100 \mathrm{mg}, \mathrm{n}=3$ ), including 11 men and 11 women, with median age of 53 years. There were two dose-limiting toxicities of AMG706, represented by diarrhea $(50 \mathrm{mg})$ and deep vein thrombosis $(75 \mathrm{mg})$. These preliminary data show that coadministration of FOLFIRI, panitumumab, and AMG706 is safe, with little effect on the pharmacokinetics of AMG706 at a dose of $50 \mathrm{mg}$ (Burris et al. 2007).

\section{Ongoing studies}

Several studies are ongoing in metastatic colorectal cancer utilizing panitumumab in combination with other chemotherapeutic agents or with targeted therapy in first-, second-, and third-line treatment. One of the largest is PACCE (Panitumumab Advanced Colorectal Cancer Evaluation), which is a multicenter trial in the US in which more than 1000 patients were randomized for first-line treatment of metastatic colorectal cancer with chemotherapy
(FOLFOX or FOLFIRI) plus bevacizumab \pm panitumumab; recruitment was initiated in 2005 and was concluded in September 2006. The preliminary analysis showed a statistically significant difference in favor of the control arm, with an increase in toxicity in the panitumumab-containing arm (Anon. 2007).

\section{Patient group/population}

Panitumumab is approved in the US for the treatment of patients with EGFR-positive metastatic colorectal cancer with disease progression on, or following, fluoropyrimidine-, oxaliplatin-, and irinotecan-containing chemotherapy. The drug is not approved in the EU.

Panitumumab is a fully human monoclonal antibody that targets the extracellular domains of EGFR. To date in all trials with panitumumab EGFR immunohistochemistry expression was assessed. Response to treatment was seen in patients with metastatic colorectal cancer with both low and negative EGFR levels (Hecht et al. 2006a, 2007). This retrospective analysis confirmed that skin rash could correlate with clinical response but not with survival. However, despite this finding, regulatory authorities have not reached a decision that could restrict continued dosing with panitumumab in patients who do not experience skin rash. Rash associated with panitumumab is common and can be classified according to the clinical dermatological manifestations, requiring specific treatment strategies (Mitchell et al. 2007).

\section{Clinical potential}

Within the last few years the natural history of metastatic colorectal cancer treatment has changed, with a dramatic improvement in OS especially with the combined modality therapy of conventional chemotherapy plus biologic agents that target a specific protein involved in tumor growth. Panitumumab has shown clear activity in metastatic colorectal cancer as a single agent and in combination chemotherapy. Panitumumab is well tolerated, with the main toxicity being skin reactions, although grade 4 severity has not been observed; no maximum tolerated dose has been reported. Because it is a fully human antibody, infusion-related reactions have been minimal; only $1 / 148$ patients experienced a grade 3 reaction and 1/463 patients discontinued treatment due to grade 2 hypersensitivity reaction in clinical trials conducted to date. Another potential advantage of panitumumab lies in its relative ease of use, with pharmacokinetic parameters unaffected by administration every week, or every 2 or 3 weeks.

Encouraging results from trials reported so far demonstrate a potential therapeutic value of this agent, but the data need to be confirmed in large multicenter trials that explore the role of panitumumab as a first- as well as a second-line option in colorectal cancer.

\section{Acknowledgements}

The authors declare that they have no conflict of interest. 


\section{References}

Anon. Amgen discontinues Vecibix(TM) treatment in PACCE trial evaluating Vectibix(TM) as part or triple combination regimen. Thousand oaks, CA: Amgen Inc. press release; March 22, 2007. Available at:

http://www.amgen.com/media_pr_detail.jsp?year=2007\&releaselD=977186 (accessed June 2007).

Berlin J, Malik I, Picus J, et al. Panitumumab therapy with irinotecan, 5 -fluorouracil, and leucovorin (IFL) in patients (pts) with metastatic colorectal cancer (mCRC). Ann Oncol. 2004;15(Suppl. 3). Abstract 265PD.

Berlin J, Neubauer M, Swanson P, et al. Panitumumab antitumor activity in patients (pts) with metastatic colorectal cancer ( $\mathrm{mCRC}$ ) expressing $\geq 10 \%$ epidermal growth factor receptor (EGFr). J Clin Oncol. 2006;24. Abstract 3548.

Burris HA, Stephenson J, Hurwitz H, et al. Safety and pharmacokinetics of AMG706 with panitumumab and FOLFIRI for the treatment of patients with metastatic colorectal cancer. ASCO 2007 Gastrointestinal Cancers Symposium. 2007. Abstract 354

Chung KY, Shia J, Kemeny NE, et al. Cetuximab shows activity in colorectal cancer patients with tumors that do not express the epidermal growth factor receptor by immunohistochemistry. J Clin Oncol. 2005;23:1803-1810.

Ciardiello F, Tortora G. A novel approach in the treatment of cancer: targeting the epidermal growth factor receptor. Clin Cancer Res. 2001;7:2958-2970.

Cunningham D, Humblet Y, Siena S, et al. Cetuximab monotherapy and cetuximab plus irinotecan in irinotecan-refractory metastatic colorectal cancer. $\mathrm{N} \mathrm{Engl} \mathrm{J} \mathrm{Med.}$ 2004;351:337-345.

de Gramont A, Bosset JF, Milan C, et al. Randomized trial comparing monthly low-dose leucovorin and fluorouracil bolus with bimonthly high-dose leucovorin and fluorouracil bolus plus continuous infusion for advanced colorectal cancer: a French intergroup study. J Clin Oncol. 1997;15:808-815.

de Gramont A, Figer A, Seymour M, et al. Leucovorin and fluorouracil with or without oxaliplatin as first-line treatment in advanced colorectal cancer. J Clin Oncol. 2000;18:2938-2947.

Dìaz Rubio E, Tabernero J, van Cutsem E, et al. Cetuximab in combination with oxaliplatin/5-fluorouracil (5-FU)/folinic acid (FA) (FOLFOX-4) in the first-line treatment of patients with epidermal growth factor receptor (EGFR)-expressing metastatic colorectal cancer: an international phase II. J Clin Oncol. 2005;23(Suppl.). Abstract 3535.

Giantonio B, Catalano PJ, Meropol NJ, et al. High-dose bevacizumab improves survival when combined with FOLFOX4 in previously treated advanced colorectal cancer: results from the Eastern Cooperative Oncoloy Group (ECOG) study E3200. J Clin Oncol. 2005;23(Suppl.). Abstract 2.

Hecht JR, Patnaik A, Malik I, et al. ABX-EGF monotherapy in patients with metastatic colorectal cancer (mCRC): an updated analysis. J Clin Oncol. 2004;22(Suppl.). Abstract 3511.

Hecht JR, Mitchell E, Baranda J, et al. Panitumumab antitumor activity in patients (pts) with metastatic colorectal cancer ( $\mathrm{mCRC}$ ) expressing low (1-9\%) or negative $(<1 \%)$ levels of epidermal growth factor receptor (EGFR). J Clin Oncol. 2006a;24(Suppl.). Abstract 3547.

Hecht JR, Posey S, Tchekmedyan E, et al. Panitumumab in combination with 5-fluorouracil, leucovorin, and irinotecan (IFL) or FOLFIRI for first-line treatment of metastatic colorectal cancer (mCRC). ASCO 2006 Gastrointestinal Cancers Symposium. 2006b. Abstract 237.

Hecht JR, Mitchell EP, Baranda J, et al. Panitumumab activity in metastatic colorectal cancer ( $\mathrm{mCRC}$ ) patients (pts) with low or negative tumor epidermal growth factor receptor (EGFr) levels: an updated analysis. ASCO 2007 Gastrointestinal Cancers Symposium. 2007. Abstract 350.

Hoff PM, Ansari R, Batist G, et al. Comparison of oral capecitabine versus intravenous fluorouracil plus leucovorin as first-line treatment in 605 patients with metastatic colorectal cancer: results of randomised phase III study. J Clin Oncol. 2001;19:2282-2292.

Hurwitz H, Fehrenbacher L, Novotny W, et al. Bevacizumab plus irinotecan, fluorouracil, and leucovorin for metastatic colorectal cancer. $N$ Engl J Med. 2004;350:2335-2342.
IARC (International Agency for Research on Cancer). GLOBOCAN 2002. IARC: Lyon; 2002. Available at: http://www-dep.iarc.fr (accessed April 3, 2007).

Jemal A, Murray T, Ward E, et al. Cancer statistics, 2005. CA Cancer J Clin. 2005;5S:10-30.

Malik I, Hecht JR, Patnaik A, et al. Safety and efficacy of panitumumab monotherapy in patients with metastatic colorectal cancer (mCRC). J Clin Oncol. 2005;23(Suppl.). Abstract 3520.

Mendelsohn J, Baselga J. The EGF receptor family as targets for cancer therapy. Oncogene. 2000;19:6550-6565.

Mendez MJ, Green LL, Corvalan JR, et al. Functional transplant of megabase human immunoglobulin loci recapitulates human antibody response in mice. Nat Genet. 1997;15:146-156.

Meyerhardt JA, Meyer RJ. Systemic therapy for colorectal cancer. $\underline{N \text { Engl J Med. }}$ 2005;352:476-487.

Mitchell EP, Rose L, Ramirez M, et al. Dermatological toxicities of panitumumab in the treatment of patients with metastatic colorectal cancer from two clinical studies. ASCO 2007 Gastrointestinal Cancers Symposium. 2007. Abstract 449.

Peters RK, Pike MC, Garabrant D, et al. Diet and colon cancer in Los Angeles County, California. Cancer Causes Control. 1992;3:457-473.

Ransohoff DF, Lang CA. Screening for colorectal cancer. N Engl J Med. 1991;325:37-41.

Saltz LB, Cox JV, Blanke C, et al. Irinotecan plus fluorouracil and leucovorin for metastatic colorectal cancer. Irinotecan Study Group. N Engl J Med. 2000;343:905-914.

Saltz LB, Rubin M, Hochster H, et al. Cetuximab (IMC-C225) plus irinotecan (CPT-11) is active in CPT-11-refractory colorectal cancer (CRC) that expresses epidermal growth factor receptor (EGFR). Proc Am Soc Clin Oncol. 2001;20. Abstract 7.

Saltz LB, Meropol NJ, Loehrer PJ, et al. Phase II trial of cetuximab in patients with refractory colorectal cancer that expresses the epidermal growth factor receptor. J Clin Oncol. 2004;22:1201-1208.

Saltz LB, Lenz H, Hochster H, et al. Randomized phase II trial of cetuximab/bevacizumab/irinotecan (CBI) versus cetuximab/bevacizumab (CB) in irinotecan-refractory colorectal cancer. J Clin Oncol. 2005;23(Suppl.). Abstract 3508.

Simmonds PC. Palliative chemotherapy for advanced colorectal cancer: systematic review and meta-analysis. Colorectal Cancer Collaborative Group. BMJ. 2000;321:531-535.

Tournigand C, Andre T, Achille E, et al. FOLFIRI followed by FOLFOX6 or the reverse sequence in advanced colorectal cancer: a randomized GERCOR study. J Clin Oncol. 2004;22:229-237.

Van Cutsem E, Hoff PM, Harper P, et al. Oral capecitabine vs intravenous 5-fluorouracil and leucovorin: integrated efficacy data and novel analyses from two large, randomised, phase III trials. Br J Cancer. 2004;90:1190-1197.

Van Cutsem E, Peeters M, Siena S, et al. Open-label phase III trial of panitumumab plus best supportive care alone in patients with chemotherapyrefractory metastatic colorectal cancer. J Clin Oncol. 2007;25:1658-1664.

Vogelstein B, Fearon ER, Hamilton SR, et al. Genetic alterations during colorectal-tumor development. N Engl J Med. 1988;319:525-532.

Weiner LM, Belldegrun A, Rowinsky E, et al. Updated results from a dose and schedule study of panitumumab (ABX-EGF) monotherapy, in patients with advanced solid malignancies. J Clin Oncol. 2005;23(Suppl.). Abstract 3059.

Winawer SJ, Shike M. Dietary factors in colorectal cancer and their possible effects on earlier stages of hyperproliferation and adenoma formation. J Natl Cancer Inst. 1992;84:74-75.

Woodburn JR. The epidermal growth factor receptor and its inhibition in cancer therapy. Pharmacol Ther. 1999;82:241-250.

Yarden Y. The EGFR family and its ligands in human cancer signalling mechanisms and therapeutic opportunities. Eur J Cancer. 2001;37:S3-S8. 
Panitumumab | Panitumumab proof of concept review

Yarden Y, Sliwkowski MX. Untangling the ErbB signalling network. Nat Rev Mol Cell Biol. 2001;2:127-137.

Correspondence: Fortunato Ciardiello, Cattedra di Oncologia Medica, Dipartimento Medico-Chirurgico di Internistica Clinica e Sperimentale "F. Magrassi e A. Lanzara", Seconda Università degli Studi di Napoli, Via S. Pansini, 5 - 80131 Napoli, Italy or at fortunato.ciardiello@unina2.it 